\title{
SIGNATURE OF LINKS
}

\author{
BY \\ LOUIS H. KAUFFMAN AND LAURENCE R. TAYLOR
}

\begin{abstract}
Let $L$ be an oriented tame link in the three sphere $S^{3}$. We study the Murasugi signature, $\sigma(L)$, and the nullity, $\eta(L)$. It is shown that $\sigma(L)$ is a locally flat topological concordance invariant and that $\eta(L)$ is a topological concordance invariant (no local flatness assumption here). Known results about the signature are re-proved (in some cases generalized) using branched coverings.
\end{abstract}

0 . Introduction. Let $L$ be an (oriented) tame link of multiplicity $\mu$ in the three-sphere $S^{3}$. That is, $L$ consists of $\mu$ oriented circles $K_{1}, \ldots, K_{\mu}$ disjointly imbedded in $S^{3}$. Various authors have investigated a numerical invariant, the signature of $L$ (notation: $\sigma(L)$ ). The signature was first defined for knots $(\mu=1)$ by $H$. Trotter [21]. J. Milnor found another definition for this knot signature (see [12]) in terms of the cohomology ring structure of the infinite cyclic cover of the knot complement. In [2], D. Erle showed that the definitions of Milnor and Trotter are equivalent. In [15], K. Murasugi formulated a definition of signature for arbitrary links.

In this paper we investigate the Murasugi signature in the context of branched covering spaces. To be specific, let $D^{4}$ denote the four dimensional ball with $\partial D^{4}=S^{3}$, and let $L \subset S^{3}$ be a link and $F \subset D^{4}$ a properly imbedded, orientable, locally flat surface with $\partial F=L \subset S^{3}$. Let $M$ denote the double branched cover of $D^{4}$ along $F$. Then we show that $\sigma(L)$ is the signature of the four manifold $M$ (see Lemma 1.1 and Theorem 3.1). Our proof of Theorem 3.1 contains the technicalities necessary to show this in the topological category. Using this viewpoint we are able to prove that $\sigma(L)$ is a topological concordance invariant (Theorem 3.8). We also rederive many of Murasugi's results, generalizing some of them (see Theorems 3.9-3.16).

The paper is organized as follows: $\S 1$ contains the classical definitions of the signature and nullity of a link. It also deals with necessary background concerning branched coverings.

Received by the editors November 4, 1974 and, in revised form, December 4, 1974 and April 11, 1975.

AMS (MOS) subject classifications (1970). Primary 55A25.

Key words and phrases. Signature, link, nullity, branched covering, concordance, isotopy. 
$\S 2$ contains theorems about the nullity of a link. The nullity is one plus the first Betti number of the double branched cover of $S^{3}$ with branch set all of $L$; it is denoted $\eta(L)$. We show that nullity is an invariant of link concordance and isotopy (Theorem 2.6). The section also contains a useful lemma about double coverings (Lemma 2.8).

$\S 3$ discusses the link signature. We show that $\sigma(L)$ may be computed from various double branched covers (Theorem 3.1) and that it is independent of the choice of spanning surface used in its definition. A number of specific results about signature are collected here. One key to our approach rests in Theorem 3.4 which gives the basic estimate for our inequalities.

$\S 4$ contains examples.

1. Definitions and branched covers. The three-sphere, $S^{3}$, will be given a fixed orientation throughout.

Let $L$ be a tame (i.e., locally flat) oriented link imbedded in $S^{3}$. Choose an orientable spanning surface $F$ for $L$; orient $F$ so that $\partial F=L$ with the chosen orientation.

The Seifert pairing $\theta: H_{1}(F) \times H_{1}(F) \rightarrow Z$ is defined by the formula $\theta(a, b)=l\left(i_{*} a, b\right)$. Here $l($,$) denotes linking number in S^{3}$, and $i_{*}$ denotes the operation of pushing into the complement of $F$ along the positive normal direction. Define a pairing $f: H_{1}(F) \times H_{1}(F) \rightarrow Z$ by the formula

$$
f(a, b)=\theta(a, b)+\theta(b, a) .
$$

This symmetrization of the Seifert pairing is bilinear, and hence it has a well-defined signature.

Definition. The signature of $L$ is defined by the equation $\sigma(L)=$ signature of $f$.

This is essentially the Murasugi definition. In [15] Murasugi takes $F$ to be a Seifert surface. This is a surface constructed by Seifert's algorithm (see [17]) from a projection of the link on $S^{2}$. He then associates a matrix $M$ with this surface and defines $\sigma(L)=\operatorname{signature}\left(M+M^{t}\right)$. One can verify that $M$ is the matrix of $\theta$ with respect to an appropriate choice of basis for $H_{1}(F)$ (see [7, pp. 37-50]). We allow an arbitrary spanning surface in our definition, but this will be seen to be legitimate in $\S 3$.

A few remarks about branched coverings will allow the first reformulation of this definition of signature.

Let $N$ be any oriented manifold with boundary such that $H_{1}\left(N ; Z_{2}\right)=0$. Let $F$ be any orientable codimension two locally-flat submanifold such that

$$
[F, \partial F]=0 \in H_{n-2}\left(N, \partial N ; Z_{2}\right) \quad(n=\operatorname{dim}(N)) .
$$

Consider the relative sequence for the pair $(N, N-F)$. 


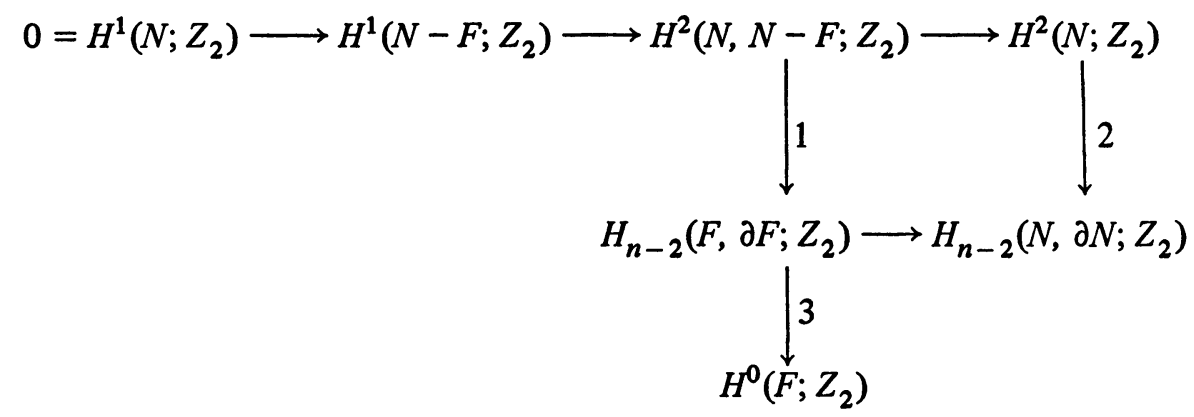

The maps 1, 2 and 3 are isomorphisms induced by Alexander duality (1) and Lefschetz duality (2 and 3). Thus $H^{1}\left(N-F ; Z_{2}\right) \cong H^{0}\left(F ; Z_{2}\right)$. Choose $\alpha \in$ $H^{1}\left(N-F ; Z_{2}\right)$ so that $\alpha$ corresponds, under this isomorphism to the sum of the generators of $H^{0}\left(F ; Z_{2}\right)$. Note that $\alpha$ is independent of the orientation choice for $F$. Let $\widetilde{N-F}$ be the two-fold covering space of $N-F$ determined by $\alpha$.

Let $\widetilde{N}=\widetilde{N-F} \cup F$, and topologize it as follows. Let $\pi: \widetilde{N} \rightarrow N$ be defined by $\pi(x)=p(x)$ for $x \in \overparen{N-F}$ and $\pi(x)=x$ for $x \in F$. Here $p: \widetilde{N-F} \rightarrow$ $N-F$ is the covering map. Let $\widetilde{N}$ have the topology generated by the sets $\pi^{-1}(U)$ where $U$ is open in $N-F$, and $p^{-1}(V \cap(N-F)) \cup(V \cap F)$ where $V$ is a connected open set in $N$ having nonempty intersection with $F$. Then the map $\pi$ is continuous, and it is clear from the local-flatness of $F$ that $\widetilde{N}$ is a topological manifold. The manifold $\widetilde{N}$ will be referred to as the double branched cover of $N$ along $F$; it will be oriented so that $\pi$ has degree +2 .

LEMMA 1.1. Let $N=D^{4}$ and let $F \subset N$ be the result of pushing a spanning surface for the link $L$ into $D^{4}$ so that $\partial F=L, F \cap S^{3}=L$. Then $\sigma(L)=$ $\sigma(\widetilde{N})$. Here $\sigma(\widetilde{N})$ denotes the signature of the middle dimensional cup-product form on $H^{2}(\widetilde{N}, \partial \widetilde{N})$.

Proof. This follows from Proposition 5.2 of [8] where it is shown that the intersection form on $\mathrm{H}_{2}(\widetilde{N})$ has matrix $V+V^{t}$ where $V$ is the matrix of the Seifert pairing $\theta$ with respect to an appropriate basis.

This lemma is the key to our approach to the link signature in $\S 3$.

Another useful invariant of links is the nullity. Let $L \subset S^{3}$ be a tame, oriented link. Let $M$ denote the double branched cover of $S^{3}$ along $L$. The nullity of $L$ is defined by the formula $\eta(L)=\beta_{1}(M)+1$. Here $\beta_{1}$ denotes the first Betti number.

It follows from Lemma 1.1 and the homology sequence for the pair $(N, \partial N)$ that $\eta(L)=\operatorname{nullity}\left(V+V^{t}\right)+1$, for $V$ the Seifert matrix for a connected spanning surface. This shows that our definition of nullity coincides with that of Murasugi.

REMARK 1.2. It follows from Proposition 5.2 of [8] that if we take even a disconnected spanning surface for $L$ in $S^{3}$, and let $N$ be the double branched 
cover of $D^{4}$ along the pushed-in surface, then $H_{2}(N) \rightarrow H_{2}(N, \partial N)$ is given by $V+V^{t}$. Hence the signature of $V+V^{t}$ is always the signature of the link. If the spanning surface $F$ has $r$ components, then $\eta(L)=\operatorname{nullity}\left(V+V^{t}\right)+r$.

2. Nullity theorems. Our definition of the nullity provides easy proofs of many theorems. As a start, since branched covers do not depend on the orientation of either the base manifold or the branch set we obtain the following.

THEOREM 2.1. The nullity is independent of the orientation on $S^{3}$ and the orientation of the link. It is also independent of the choice of spanning surface.

If $L_{1}$ and $L_{2}$ are links in $S^{3}$, let $L_{1} \circ L_{2}$ be the link obtained from $S^{3} \# S^{3}$ with $L_{1}$ in one piece and $L_{2}$ in the other (disjointly). Let $L_{1} \oplus L_{2}$ be the link obtained by choosing a 3-ball, $B_{i}$, in each $S^{3}$ such that $B_{i} \cap L_{i}$ is a single strand. Form $S^{\mathbf{3}} \# S^{\mathbf{3}}$ by removing these 3-balls, and let the gluing homeomorphism $S^{2} \rightarrow S^{2}$ take the two points in $\partial B_{1} \cap L_{1}$ to the two in $\partial B_{2} \cap L_{2}$. The link $L_{1} \oplus L_{2}$ is not well defined, but we let this symbol denote any link obtained by the above procedure.

THEOREM 2.2. $\eta\left(L_{1} \circ L_{2}\right)=\eta\left(L_{1}\right)+\eta\left(L_{2}\right), \eta\left(L_{1} \oplus L_{2}\right)=\eta\left(L_{1}\right)+\eta\left(L_{2}\right)$ -1 .

The proof, which is left to the reader, consists of looking at double branched covers for $L_{1} \circ L_{2}$ and $L_{1} \oplus L_{2}$.

Let us decompose the double branched cover, $N$, of $S^{3}$ along $L$ as follows. There is $V$, a tubular neighborhood of the branch set, and $\widetilde{M}$, a double cover of $M=S^{3}-T$; here $T$ is a tubular neighborhood of $L \subset S^{3}$. Mayer-Vietoris gives

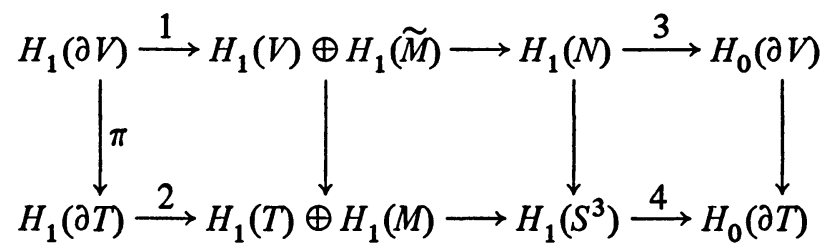

Maps 3 and 4 are easily seen to be zero. The map $\pi$ is induced from a collection of tori double covering themselves. Thus, with rational coefficients, it is an isomorphism. Hence 1 is a monomorphism since 2 clearly is. We conclude that $\eta(L)=\beta_{1}(\widetilde{M})-\mu(L)+1$.

This shows that the nullity depends only on $\widetilde{M}$, or only on $S^{3}-L$ and a homomorphism $H_{1}\left(S^{3}-L ; Z_{2}\right) \rightarrow Z_{2}$.

To compute $\eta(L)$, we could compute $H_{*}\left(\widetilde{M} ; Z_{2}\right)$ and apply the Bockstein spectral sequence. This is not as facetious as it sounds, for $H_{*}\left(\widetilde{M} ; Z_{2}\right)$ is easy to compute using the Gysin sequence. (We are indebted to Frank Connolly for suggesting the Gysin sequence approach, which greatly simplified matters.) The Gysin 
sequence reads

$$
\cdots \rightarrow H_{*+1}\left(M ; Z_{2}\right) \stackrel{\cap_{x}}{\longrightarrow} H_{*}\left(M ; Z_{2}\right) \rightarrow H_{*}\left(\widetilde{M} ; Z_{2}\right) \stackrel{\pi}{\longrightarrow} H_{*}\left(M ; Z_{2}\right) \rightarrow \cdots
$$

(e.g., Spanier $[18$, p. 260]).

In our case, using Alexander duality, $H_{1}\left(M ; Z_{2}\right) \cong Z_{2}^{\mu(L)}$ generated by meredians in $\partial T ; H_{2}\left(M ; Z_{2}\right) \cong Z_{2}^{\mu(L)-1}$ and $H_{2}\left(\partial T ; Z_{2}\right)$ maps onto it. The map $H_{2}(\partial T) \rightarrow H_{2}(M) \stackrel{\cap x}{\longrightarrow} H_{1}(M)$ is given by the matrix $L=\left(l_{i j}\right)$ where $l_{i j}=$ $l$ (ith component, $j$ th component) for $i \neq j$, and $l_{i i}=-\Sigma_{j=1 ; j \neq i}^{\mu(L)} l_{i j}$. (This may be seen by interpreting the map $\bigcap_{\chi}$ as intersection with a spanning surface for $L$.)

Since $\beta_{1}(\widetilde{M}) \leqslant \operatorname{dim} H_{1}\left(\widetilde{M} ; Z_{2}\right)$, we conclude the following.

THEOREM 2.3. $\eta(L) \leqslant$ nullity $_{Z_{2}} L$ where we take the nullity over the field $Z_{2}$.

Corollary 2.4. $1 \leqslant \eta(L) \leqslant \mu(L)$.

REMARK 2.5. The integer nullity $Z_{2} L$ is a link homotopy invariant since Milnor [13] shows $L$ is. Example (1) shows that nullity is not a link homotopy invariant.

We can now define $\eta(L)=\operatorname{dim}$ (quotient group of $H_{1}\left(\widetilde{M} ; Z_{2}\right)$ consisting of permanent cycles in the Bockstein spectral sequence) $-\mu(L)+1$. This defines a nullity even for wild links.

Two links $L_{1}$ and $L_{2}$ are said to be link concordant if there exists a topological imbedding of $F=\mu\left(L_{1}\right)$ copies of $S^{1} \times I$ in $S^{3} \times I$, such that $\partial_{-} F \subset$ $S^{3} \times 0$ is $L_{1}$ and $\partial_{+} F \subset S^{3} \times 1$ is $L_{2}$. A link $L$ is said to be null link concordant if there is an imbedding of $F_{1}=\mu(L)$ copies of $D^{2}$ in $D^{4}$ such that $\partial F_{1} \subset S^{3}$ is $L$. Neither imbedding is required to be nice in any fashion whatsoever.

THEOREM 2.6. If $L_{1}$ and $L_{2}$ are link concordant, then $\eta\left(L_{1}\right)=\eta\left(L_{2}\right)$. If $L$ is null link concordant, then $\eta(L)=\mu(L)$.

COROllary 2.7. Nullity is a link isotopy invariant (see Milnor [14]).

Proof (of 2.6). By adding a collar, we may assume our imbeddings are proper $(F \subset N$ is proper if $F \cap \partial N=\partial F)$. Let $M=S^{3} \times I-F$ with boundary components $\partial_{+} M$ and $\partial_{-} M$. Alexander duality shows $\partial_{ \pm} M \subset M$ is a mod-2 homology equivalence. The Gysin sequence shows $\partial_{ \pm} \widetilde{M} \subset \widetilde{M}$ is a mod-2 homology equivalence. The Bockstein spectral sequence shows $\eta\left(L_{1}\right)=\eta\left(L_{2}\right)$.

Now consider $D^{4}-F_{1}$. Since $H_{1}\left(D^{4}-F_{1}\right) \cong Z_{2}^{\mu(L)}$ is the only nontrivial homology group (except $H_{0}$ ), the Gysin sequence shows $H_{1}\left(\widetilde{D^{4}-F_{1}}\right) \cong Z_{2}^{2 \mu(L)-1}$ is the only nontrivial homology group. Thus, using the Gysin sequence again, $H_{1}\left(\widetilde{\left.S^{3}-L\right)} \rightarrow H_{1}\left(\widetilde{D^{4}-L}\right)\right.$ must be onto. The naturality of the Gysin sequence and the Bockstein spectral sequence shows $\eta(L)=\mu(L)$.

The next to the last sentence contains an observation we will need again. 
LeMma 2.8. Let $f: X \rightarrow Y$ induce a monomorphism on $H_{0}\left(; Z_{2}\right)$ and an epimorphism on $H_{1}\left(; Z_{2}\right)$. If $\widetilde{f}: \widetilde{X} \rightarrow \widetilde{Y}$ is a double cover, then $\widetilde{f_{*}}$ is monic on $H_{0}\left(; Z_{2}\right)$ and epic on $H_{1}\left(; Z_{2}\right)$. If $H_{1}(\tilde{Y} ; Z)$ is finitely generated, then $\widetilde{f}_{*}$ is epic on $H_{1}(; Q)$. $\left(\widetilde{f}_{*}\right.$ is the map induced by $\widetilde{f}$ on homology.)

The proof is just the relative Gysin sequence and universal coefficients.

As a final result, we state

THEOREM 2.9. $\eta(L)=1$ if and only if $\nabla_{L}(-1) \neq 0$.

This result is proved in [5, Theorem 1]. Recall [4]

$$
\nabla_{L}(t)= \begin{cases}\Delta(t), & \mu(L)=1, \\ \Delta(t, t, \ldots, t)(1-t)^{2-\mu(L)}, & \mu(L) \geqslant 2,\end{cases}
$$

where $\Delta(t, t, \ldots, t)$ is the Alexander polynomial.

3. The signature. Let $L \subset S^{3}$ be an oriented link. Let $F \subset D^{4}$ be a properly imbedded, oriented, locally flat surface with $\partial F=L$. Let $N(F)$ denote the double branched cover of $D^{4}$ along $F$. We wish to show that $\sigma(L)=\sigma(N(F))$. Thus the link signature is independent of the choice of spanning surface. In the piecewise-linear or differentiable categories, this is an easy matter: Let $F^{\prime} \subset D^{4}$ be another such surface. Let $F^{\prime \prime} \subset D^{5}$ be a properly imbedded submanifold so that $\partial F^{\prime \prime}=F \cup-F^{\prime} \subset D^{4} \cup-D^{4}=S^{4}$. Then $\partial N\left(F^{\prime \prime}\right)=N(F) \cup-N\left(F^{\prime}\right)$. Hence, by the Novikov addition theorem (see [1, p. 588]), $\sigma(N(F))=\sigma\left(N\left(F^{\prime}\right)\right)$. In particular, let $F^{\prime}$ denote a pushed-in spanning surface for $L$. Then $\sigma(L)=$ $\sigma\left(N\left(F^{\prime}\right)\right)=\sigma(N(F))$, proving the assertion.

In fact, we shall generalize this idea as follows. Let $M$ be an oriented four manifold with boundary such that $H_{1}\left(M ; Z_{2}\right)=0$. Let $F$ be any orientable codimension-two, locally-flat submanifold with $[F, \partial F]=0 \in H_{2}\left(M, \partial M ; Z_{2}\right)$. Then, as in $\S 1$, we can form $N$, the double branched cover of $M$ along $F$.

Suppose that $\partial M=S^{3}$ with $\partial F \subset S^{3}$ some link $L$. Suppose also that $[F, \partial F]=\chi \in H_{2}(M, \partial M ; Z)$, with $\chi$ divisible by 2 .

Define $\chi^{2} \in \mathrm{Z}$ as follows. Let $F_{1} \subset S^{3}$ denote a spanning surface for $\partial F \subset S^{3}$. Let $\bar{M}=M \cup D^{4}$ with the boundary three-spheres identified. Let $\bar{\chi}=\left[F-F_{1}\right] \in H_{2}(\bar{M} ; Z)$, and define $\chi^{2}=\bar{\chi}^{2}$ where $\bar{\chi}^{2}$ denotes the self-intersection number of $\bar{\chi}$.

Note that $\chi^{2}$ may also be interpreted as the linking number of $\partial F$ with $\partial F^{\prime}$ in $S^{3}$. Here $F^{\prime}$ denotes the result of pushing $F$ off itself in $M$ so that $F \cap F^{\prime}=$ $\varnothing$ and $F^{\prime} \cap \partial M=\partial F^{\prime}$.

The fundamental observation of this section is the following result.

THEOREM 3.1. $\sigma(L)=\sigma(N)-2 \sigma(M)+1 / 2 \chi^{2}$ where the double branched cover $N$ is oriented so that $\pi: N \rightarrow M$ has degree +2 . 
Proof. If $F \subset M$ is a smooth imbedding, we proceed as follows. Let $B$ be the double branched cover of $D^{4}$ along some pushed-in surface for $L$. Thus $\sigma(L)=\sigma(B)$ by Lemma 1.1. Now Proposition 6.15 of [1] (see [6] for an elementary proof) shows $\sigma(N)-\sigma(B)=2 \sigma(M)-\chi^{2} / 2$ from which our result follows. The same result is still true for locally-flat imbeddings [19]. We will close this section with a proof of 3.1 for $\chi=0$, in the topological category.

REMARK 3.2. Note that 3.1 shows that $\sigma(L)$ is independent of the choice of spanning surface. If $F$ is any locally-flat, orientable surface in $D^{4}$ with $\partial F=$ $L \subset S^{3}$, then $\sigma(L)=\sigma(N)$ where $N$ is the double branched cover of $D^{4}$ along $F$.

Given a link $L \subset S^{3}$, let $\lambda(L)=\Sigma_{1 \leqslant i<j \leqslant \mu(L)} l\left(K_{i}, K_{j}\right)$ where $L=K_{1} \cup$ $K_{2} \cup \cdots \cup K_{\mu}$. Here is a proof of the following theorem due to Murasugi [15]. From our approach, one sees that the result depends only on the fact that double branched covers are independent of orientations.

THEOREM 3.3. $\sigma(L)+\lambda(L)$ is independent of the orientations of the components of $L$.

Proof. Let each component $K_{i}$ bound $F_{i} \subset D^{4}$. We can always take the $F_{i}$ so that $F_{i} \cap F_{j} \cap F_{k}=\varnothing$ for $i, j, k$ distinct, and so that $F_{i} \cap F_{j}$ is exactly $\left|l\left(K_{i}, K_{j}\right)\right|$ points.

Let $B^{4} \subset D^{4}$ be a small ball about a point in $F_{i} \cap F_{j}$. We can insist that $\left(F_{i} \cap \partial B^{4}\right) \cup\left(F_{j} \cap \partial B^{4}\right) \subset \partial B^{4}$ be either $O$ or $O($ see Wall [22]). The first link bounds two disjoint disks in $C P^{2}-B^{4}$ such that $\left[D_{1}\right]=-\left[D_{2}\right]$ in $H_{2}\left(C P^{2}-B^{4}\right)$. The other does the same except in $\left(-C P^{2}\right)-B^{4}$.

Let $M$ denote the connected sum of $D^{4}$ and a collection of copies of $C P^{2}$ and $-C P^{2}$, one for each intersection point. $M$ is obtained by adding a $\pm C P^{2}$ at each crossing of $F_{i} \cap F_{j}$ and then removing the double point. This gives $F \subset M$ with $\partial F=L \subset \partial M=S^{3}$. Then $[F, \partial F]=0 \in H_{2}(M, M ; Z)$.

Hence $\sigma(L)=\sigma(N)-2 \sigma(M)$.

Let $L^{*}$ be the link obtained by reorienting one component, say $K_{1}$. Then, for the new surface $F^{*}$ bounding $L^{*}$ we have $\left[F^{*}, \partial F^{*}\right]=2 \chi$. It is not hard to see $\chi^{2} \doteq-\Sigma_{1<j \leqslant \mu(L)} l\left(K_{1}, K_{j}\right)$. (Here $K_{1}$ has the new orientation.) Hence $\sigma\left(L^{*}\right)=\sigma(N)-2 \sigma(M)-2 \Sigma l\left(K_{1}, K_{j}\right)$, or $\sigma\left(L^{*}\right)=\sigma(L)-2 \Sigma l\left(K_{1}, K_{j}\right)$, or $\sigma\left(L^{*}\right)$ $+\lambda\left(L^{*}\right)=\sigma(L)+\lambda(L)$, proving the theorem.

The following situation gives our remaining theorems. Let $M$ be a 4-manifold with $H_{1}\left(M ; Z_{2}\right)=0$ and $\partial M$ a collection of copies of $S^{3}$. In practice, $M$ is $D^{4}, S^{3} \times I, S^{4}$, or $S^{3} \times I-D^{4}$. Let each component of $\partial M$ contain a link $L_{i}$, and let $F \subset M$ be a surface with $\partial F=\bigcup_{i} L_{i}$. Let $N$ be the double branched cover of $M$ along $F$. By the Novikov addition theorem, $\sigma(N)=\Sigma_{i=1}^{\beta}{ }^{(\partial M)} \sigma\left(L_{i}\right)$. Now $\sigma(N)$ is also the signature of the cup product form on $H^{2}(N, \partial N ; Q)$. In 
general, this form is singular, but if $K$ is the cokernel of $H^{1}(\partial N ; Q) \rightarrow$ $H^{2}(N, \partial N ; Q)$, then the form restricts to a nonsingular form on $K$ with the same signature. Hence $|\sigma(N)| \leqslant \operatorname{dim} K$ and $\sigma(N) \equiv \operatorname{dim} K(\bmod 2)$.

THEOREM 3.4. The dimension of $K$ is given by

$$
\operatorname{dim} K=2 \chi(M)-\chi(F)+2 \beta_{1}(N)-\sum_{i=1}^{\beta_{0}(\partial M)} \eta\left(L_{i}\right)+2 \beta_{0}(\partial M)-2 .
$$

Corollary 3.5. If $L \subset S^{3}$, is a link, then $\sigma(L) \equiv \eta(L)+\mu(L)(\bmod 2)$.

Proof. Write down the cohomology sequence of the pair $(N, \partial N)$ beginning with $0 \rightarrow H^{0}(N)$ and ending with $H^{2}(N, \partial N) \rightarrow K \rightarrow 0$. Then $\operatorname{dim} K$ is an alternating sum of various Betti numbers. The following formulas are useful: $2 \chi(M)-\chi(F)=\chi(N), \beta_{2}(N, \partial N)=\beta_{2}(N), \beta_{1}(N, \partial N)=\beta_{3}(N), \beta_{1}(\partial N)=\Sigma_{i} \eta\left(L_{i}\right)$ $-\beta_{0}(\partial M), \beta_{0}(\partial N)=\beta_{0}(\partial M)$. The proof is now obvious. To get 3.5, apply 3.4 to any orientable surface in $D^{4}$ spanning $L$.

Thus we need ways of computing or estimating $\beta_{1}(N)$.

THEOREM 3.6. Let $\partial_{i} M$ be the component of $\partial M$ containing $L_{i}$ and let $\partial_{i} F=F \cap \partial_{i} M$. If $F$ is orientable, and if $H_{0}\left(\partial_{i} F\right) \rightarrow H_{0}(F)$ is onto, then $\beta_{1}(N) \leqslant \eta\left(L_{i}\right)-1$.

Proof. Use Alexander duality to compute $H_{1}\left(M-F, \partial_{i} M-\partial_{i} F ; Z_{2}\right)=0$. Use Lemma 2.8 to show $H_{1}\left(\widehat{M-F}, \widetilde{\partial_{i} M-\partial_{i} F}\right)=0$, first with $Z_{2}$ coefficients and thus also with $Q$. Use Alexander duality again to show $H_{1}\left(N, \partial_{i} N ; Q\right)=0$. This completes the proof.

THEOREM 3.7. Let $F \subset M$ as above have $\beta_{0}(F)$ components. Then $\beta_{1}(N) \leqslant$ $\beta_{0}(F)-1$.

Proof. Using Mayer-Vietoris, it is sufficient to show $\beta_{1} \overline{(\overline{M-F})} \leqslant 2 \beta_{0}(F)$ -1. Let $\Lambda$ be a wedge of $\beta_{0}(F)$ circles. There is a map $\Lambda \rightarrow M-F$ which is onto in $H_{1}\left(; Z_{2}\right)$, as is not hard to see. By Lemma $2.8, \widetilde{\Lambda} \rightarrow \widetilde{M-F}$ is also onto in $H_{1}(; Q)$. But $\beta_{1}\left(\widetilde{\mathcal{N}}=2 \beta_{0}(F)-1\right.$ as is well known. This completes the proof.

THEOREM 3.8. Signature is a topological concordance invariant.

Proof. Let $L_{1}$ and $L_{2} \subset S^{3}$ be concordant; that is, there is a locally-flat imbedding of $F=\mu\left(L_{1}\right)$ copies of $S^{1} \times I$ in $S^{3} \times I$ with $\partial_{+} F=L_{2}$ and $\partial_{-} F=$ $-L_{1}$, the mirror image of $L_{1}$. It is easy to see $\sigma\left(-L_{1}\right)=-\sigma\left(L_{1}\right) . N$ is the double branched cover. By Theorem 3.6, $\beta_{1}(N) \leqslant \eta\left(L_{1}\right)-1$ and $\eta\left(L_{2}\right)-1$. Theorem 3.4 shows $\operatorname{dim} K \leqslant-\left|\eta\left(L_{1}\right)-\eta\left(L_{2}\right)\right|$. Thus $\eta\left(L_{1}\right)=\eta\left(L_{2}\right)$ (which also follows from 2.6) and $\sigma(N)=0$. Hence $\sigma\left(L_{1}\right)=\sigma\left(L_{2}\right)$. 
THEOREM 3.9. Let $F$ be an orientable surface in $D^{4}$ spanning $L \subset S^{3}$, and assume $F$ has no closed components. Then

$$
|\sigma(L)| \leqslant 2 \operatorname{genus}(F)-\mu(L)-\max \left(\eta(L), 2 \beta_{0}(F)-\eta(L)\right) .
$$

Corollary 3.10 (Murasugi [15, Theorem 9.1]).

$$
|\sigma(L)| \leqslant 2 \text { genus }(F)+\mu(L)-\beta_{0}(F) .
$$

Corollary 3.11. Suppose $\nabla_{L}(-1) \neq 0$. Then

$$
|\sigma(L)| \leqslant 2 \text { genus }(F)+\mu(L)+1-2 \beta_{0}(F) .
$$

COROLlary 3.12. If $g^{*}(L)$ is the minimal genus of an oriented connected surface in $D^{4}$ spanning $L$, then

$$
|\sigma(L)| \leqslant 2 g^{*}(L)+\mu(L)-\eta(L) .
$$

Proof. Apply Theorem 3.4. Estimate $\beta_{1}(N)$ once using Theorem 3.6 and again using 3.7. The corollaries are obvious.

Now let $L \subset S^{3}$ be a link, and let $S^{3} \subset S^{4}$ be the standard imbedding. There exist closed locally-flat surfaces $P \subset S^{4}$ such that $P \cap S^{3}=L$ and every component of $P$ meets $S^{3}$. Define the slice genus, $g_{s}(L)$, as the minimum genus of such an oriented surface. Since $S^{3}$ divides $S^{4}$ into two components, it also divides $P$ into surfaces $F_{1}$ and $F_{2}$. Let $h(L)$ be the minimum of $\beta_{0}\left(F_{1}\right)-\beta_{0}(P)$ over all $P \subset S^{4}$ such that $P \cap S^{3}=L$, each component of $P$ meets $S^{3}$, and $\operatorname{genus}(P)=g_{s}(L)$.

A slice link is one for which $g_{s}(L)=0$, and a slice link in the strong sense is one for which $g_{s}(L)=h(L)=0$.

THEOREM 3.13. $|\sigma(L)| \leqslant g_{s}(L)+\min \left(0, \eta(L)-\beta_{0}(P)\right)$ and $0 \leqslant$ $(\mu(L)-\eta(L)) / 2 \leqslant 2 g_{s}(L)+h(L)$.

Proof. Let $N$ be the double branched cover of $S^{4}$ along $P$. Let $W_{1}$ and $W_{2}$ be the two pieces of $N$ mapping onto the two pieces of $S^{4}-S^{3}$. Thus, $\partial W_{1}=\partial W_{2}=M$, the double branched cover of $S^{3}$ along $L$. The sequence $H^{2}\left(W_{i}, M ; Q\right) \rightarrow H^{2}(N ; Q) \rightarrow H^{2}\left(W_{i} ; Q\right)$ and Mayer-Vietoris show $\operatorname{dim} K_{1}+$ $\operatorname{dim} K_{2} \leqslant \operatorname{dim} H^{2}(N ; Q)$ where $K_{i}$ is the cokernel of $H^{1}(M) \rightarrow H^{2}\left(W_{i}, M\right)$. Hence $|\sigma(L)| \leqslant 1 / 2 \operatorname{dim} H^{2}(N ; Q)$.

We also know that $\operatorname{dim} H^{2}(N ; Q)=-2+2 \beta_{1}(N)+\chi(N)$ and $\chi(N)=$ $4-\chi(P)=4-2 \beta_{0}(P)+2 g_{s}(L)$. By Theorem 3.7, $\beta_{1}(N) \leqslant \beta_{0}(P)-1$. One checks that $H_{1}\left(S^{3}-L ; Z_{2}\right) \rightarrow H_{1}\left(S^{4}-P ; Z_{2}\right)$ is onto, and then, that $H_{1}(M ; Q)$ $\rightarrow H_{1}(N ; Q)$ is also onto. Hence $\beta_{1}(N) \leqslant \eta(L)-1$. This gives the first formula.

To see the second formula, proceed as follows. Let $F_{i}=P \cap W_{i}$. Then $H_{1}\left(W_{i}, W_{i}-F_{i}\right)=0$ by the Thom isomorphism, so $H_{1}\left(W_{i}-F_{i}\right) \rightarrow H_{1}\left(W_{i}\right)$ is 
onto. It is now easy to see that $H_{1}\left(W_{i}-F_{i}, M-\partial F_{i}\right) \rightarrow H_{1}\left(W_{i}, M\right)$ is onto. By Lemma 2.8 , to show $H_{1}\left(W_{i}, M\right)=0$, it is enough to show $H_{1}\left(D^{4}-F_{i}, S^{3}-\partial F_{i}\right)$ $=0$. By Alexander duality this group is isomorphic to $H^{3}\left(D^{4}, F_{i}\right)$, which is 0 . This shows $\beta_{3}\left(W_{i}\right)=0$ and $\beta_{1}(M) \geqslant \beta_{1}\left(W_{i}\right)$.

Next show $\beta_{1}\left(W_{i}\right) \geqslant \chi\left(F_{i}\right)-1$. Estimate $\chi\left(F_{i}\right)=\beta_{0}\left(F_{i}\right)-\beta_{1}\left(F_{i}, \partial F_{i}\right)$ using the excision $\beta_{1}\left(F_{i}, \partial F_{i}\right)=\beta_{1}\left(P, \partial F_{i^{\prime}}\right)$ so as to show

$$
\beta_{1}(M) \geqslant\left|\beta_{0}\left(F_{1}\right)-\beta_{0}\left(F_{2}\right)\right|+\beta_{0}(P)-\beta_{1}(P)-1 .
$$

Since $P=F_{1} \cup F_{2}$,

$$
\beta_{0}\left(F_{1}\right)+\beta_{0}\left(F_{2}\right) \geqslant \mu(L)-\beta_{1}(P)+\beta_{0}(P),
$$

from which the required formula follows easily.

Corollary 3.14. If $L$ is a slice link, $\sigma(L)=0$. If moreover, $L$ is a slice link in the strong sense, then $\eta(L)=\mu(L)$.

REMARK 3.15. If $F \subset D^{4}$ spans $L$, then $g_{s}(L) \leqslant 2$ genus $(F)+\mu(L)-\beta_{0}(F)$, so we recover 3.10 from 3.13 .

We next wish to consider the effect of the following operation. Let $L_{1} \subset$ $S^{3}$ be a link and let $D^{3} \subset S^{3}$ intersect $L_{1}$ in a two stranded braid $b_{1}$. Both strings of $b_{1}$ are oriented. Let $b_{2}$ be another two stranded braid with the same orientation as $b_{1}$, and let $L_{2}$ be the link obtained by replacing $b_{1}$ with $b_{2}$. Two examples of this operation are: (1) change an overcrossing to an undercrossing; and (2) add two components of a link together.

A two stranded braid may be visualized as in Figure 1 and is classified by the number of crossings, and whether the first cross is under or over. Our braids also have an orientation.

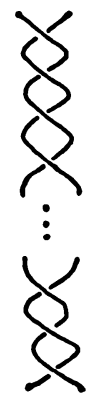

FiguRE 1

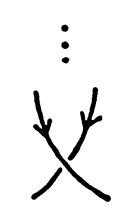

FigURE 2

Given a two stranded braid $b$, define $\sigma(b)$ as follows. If the two strands have opposite orientations, $\sigma(b)=0$. If they have the same orientation, $\sigma(b)=$ 
$\epsilon \cdot(N-1)$ where $N$ is the number of crossings, $\epsilon= \pm 1$, and $\epsilon=+1$ if the braid ends as in Figure 2 (that is, a left-right overcrossing in the direction of the orientations).

THEOREM 3.16. With notation as above,

$$
\left|\sigma\left(L_{1}\right)-\sigma\left(b_{1}\right)-\left(\sigma\left(L_{2}\right)-\sigma\left(b_{2}\right)\right)\right|+\left|\eta\left(L_{1}\right)-\eta\left(L_{2}\right)\right|+\left|\mu\left(L_{1}\right)-\mu\left(L_{2}\right)\right| \leqslant 3 .
$$

Modulo 2, the left-hand side is congruent to $\sigma\left(b_{1}\right)-\sigma\left(b_{2}\right)$. Furthermore,

$$
\left|\eta\left(L_{1}\right)-\eta\left(L_{2}\right)\right| \leqslant 1 \text { and }\left|\mu\left(L_{1}\right)-\mu\left(L_{2}\right)\right| \leqslant 1 \text {. }
$$

We defer the proof momentarily, and discuss some consequences.

Given two arcs in a link, we may isotop them into a disc $D^{3}$ in such a way that the braid is trivial and the two strands have the same orientation. We may replace this braid by either one of two braids with a single crossing. Call either of these the link obtained by adding either two components of our original link, or one component to itself.

Corollary 3.17. Let $L_{1}$ be a link and let $L_{2}$ be a link obtained by adding two components of $L_{1}$. Then

$$
\left|\sigma\left(L_{2}\right)-\sigma\left(L_{1}\right)\right|+\left|\eta\left(L_{2}\right)-\eta\left(L_{1}\right)\right| \leqslant 1 .
$$

Corollary 3.18. $\sigma\left(L_{1} \oplus L_{2}\right)=\sigma\left(L_{1}\right)+\sigma\left(L_{2}\right), \sigma\left(L_{1} \circ L_{2}\right)=\sigma\left(L_{1}\right)+$ $\sigma\left(L_{2}\right)$

CoROLLARY 3.19. If $L_{1}$ is a link and $L_{2}$ is the link obtained by adding two components of $L_{1}$, and if $\eta\left(L_{1}\right)=\mu\left(L_{1}\right)$, then

$$
\eta\left(L_{2}\right)=\mu\left(L_{2}\right) \text { and } \sigma\left(L_{1}\right)=\sigma\left(L_{2}\right) .
$$

Corollary 3.20. Let $L_{1}$ be a link and $L_{2}$ be the link obtained from $L_{1}$ by changing an overcrossing in a projection of $L_{1}$ to an undercrossing. Then $\left|\sigma\left(L_{2}\right)-\sigma\left(L_{1}\right)\right|+\left|\eta\left(L_{2}\right)-\eta\left(L_{1}\right)\right|=0$ or 2 and $\left|\eta\left(L_{2}\right)-\eta\left(L_{1}\right)\right| \leqslant 1$.

Definition. The unlinking number, $U(L)$, of a link $L$ is the minimum number of operations of the type: change an underpass to an overpass, needed to produce the trivial link on $\mu(L)$ components.

COROLlaRY 3.21. $|\sigma(L)|+\mu(L)-\eta(L) \leqslant 2 U(L)$ and $\mu(L)-\eta(L) \leqslant U(L)$.

PrOOF (OF 3.16). First consider the case where the braid strands have the same orientation. Then, if the braid $b$ has $N$ crossings, one can choose a spanning surface for the link so that the matrix $M=V+V^{t}$ has the form 


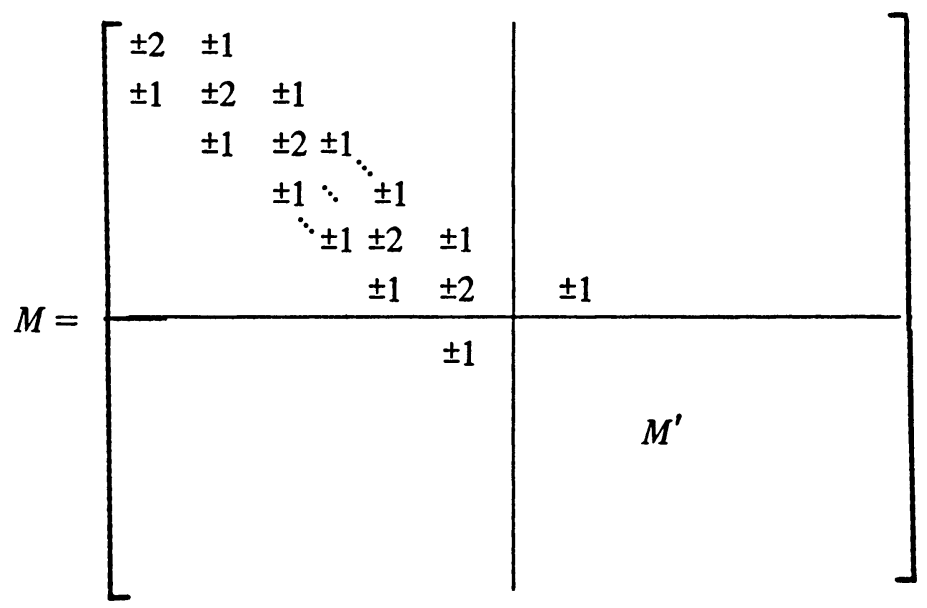

where the upper left-hand block is an $(N-1) \times(N-1)$ matrix. Braid replacement corresponds to changing the size, and possibly the sign, of this block along with the signs of the auxiliary \pm 1 entries.

Now $M$ is certainly congruent to a matrix $\bar{M}$ of the following form

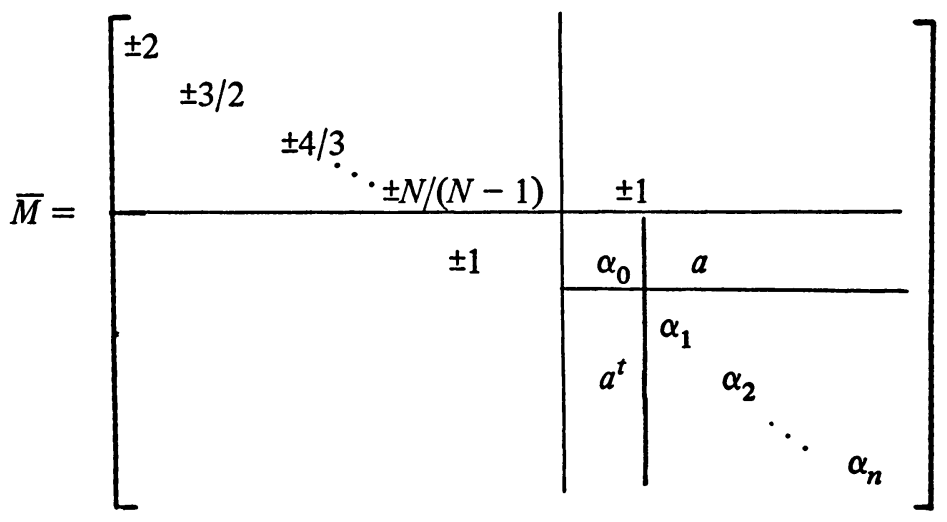

The theorem now follows by considering the effect of changing $N$ and the signs upon the signature and nullity of $\bar{M}$.

To be more precise, we see that $\sigma(\bar{M})=\sigma(b)+\sigma(\overline{\bar{M}})$ and nullity $(\bar{M})=$ nullity $(\overline{\bar{M}})$ where the matrix $\overline{\bar{M}}$ denotes the lower right-hand block of $\bar{M}$ with $\alpha_{0}$ replaced by $\alpha_{0}^{\prime}=\alpha_{0} \pm(N-1) / N$. Thus the value of $\alpha_{0}^{\prime}$ depends upon $N$ and the sign, $\epsilon$, of the braid. It is easy to check that the signature of $\overline{\bar{M}}$ can change by at most two when $\alpha_{0}^{\prime}$ is varied. If the signature of $\overline{\bar{M}}$ changes by two, then the nullity remains constant. If the signature of $\overline{\bar{M}}$ changes by zero or one, then the nullity may change by at most one. Finally, the multiplicity, $\mu(L)$, changes by no more than one, under braid replacement. We sum this up by observing that if $\overline{\bar{M}}_{1}$ is the matrix for $L_{1}$, and $\overline{\bar{M}}_{2}$ is the matrix for $L_{2}$, then $\left|\sigma\left(\overline{\bar{M}}_{1}\right)-\sigma\left(\overline{\bar{M}}_{2}\right)\right|+$ $\left|\eta\left(L_{1}\right)-\eta\left(L_{2}\right)\right|+\left|\mu\left(L_{1}\right)-\mu\left(L_{2}\right)\right| \leqslant 3$. Hence the theorem follows, since $\sigma\left(\overline{\bar{M}}_{i}\right)$ $=\sigma\left(L_{i}\right)-\sigma\left(b_{i}\right)$ for $i=1,2$.

The case where the braid strands have opposite orientation is handled similarly. 
This completes the proof of 3.16. To prove Corollary 3.18, note that signatures of four-manifolds add for boundary connected sum. Hence $\sigma\left(L_{1} \circ L_{2}\right)=$ $\sigma\left(L_{1}\right)+\sigma\left(L_{2}\right)$. Now apply 3.17 and 2.2. The other corollaries follow easily.

Finally, we give the promised proof of Theorem 3.1 in the case $\chi=0$.

Proof. We need only show that if $F$ is a locally-flat orientable surface in $M$, a closed, compact 4-manifold and if $N$ is the double branched cover of $M$ along $F$, then $\sigma(N)=2 \sigma(M)$.

$F \times R^{1} \subset M \times R^{1}$ has a normal bundle by [9], so we get a map $f: M \times$ $R^{1} \rightarrow \operatorname{MSTOP}(2)$ with $f$ transverse to BSTOP(2) and $f^{-1}(\mathrm{BSTOP}(2))=F \times R^{1}$. Let $g: M \rightarrow \operatorname{MSTOP}(2)$ be the composite of $M \rightarrow M \times 0 \subset M \times R^{1}$ with $f$. Then $g^{-1}(\mathrm{BSTOP}(2))=F$, but we do not claim $g$ is transverse to $\operatorname{BSTOP}(2)$.

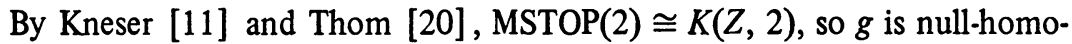
topic since $\chi=0$. Thus there is a map $H: M \times I \rightarrow \operatorname{MSTOP}(2)$ such that $H \mid M \times$ $[0, \epsilon]=f \mid M \times[0, \epsilon]$ and $H(M \times[1-\epsilon, 1])=\infty \in \operatorname{MSTOP}(2)$ for some $\epsilon$, $0<\epsilon<1 / 2$.

$H$ is transverse to $\operatorname{BSTOP}(2)$ on $M \times(0, \epsilon] \cup M \times[1-\epsilon, 1)$, so by the transverse regularity theorem [10], we can make $H$ transverse to $\operatorname{BSTOP}(2)$ on $M \times(0,1)$ rel $M \times(0, \epsilon] \cup M \times[1-\epsilon, 1)$. Hence we get $P \subset M \times I$ such that $P$ is locally-flat, $\partial P=F, P \cap M \times 1=\varnothing$, and $P \cap M \times 0=\partial P$.

It is possible to add a 0 -handle to $M \times 0 \cup M \times 1$ so that we have $M \#-M$ bounded by $W$ with $H$ becoming a map $h: W \rightarrow \operatorname{MSTOP}(2)$ such that $P=$ $h^{-1}(\mathrm{BSTOP}(2))$. Then $H_{3}(W, \partial W ; Z) \cong H_{2}(M ; Z)$ and $[P, \partial P]$ goes to $\chi$ under this map. Hence we can form the double branched cover of $W$ along $P$. This bounds $N \#-M \#-M$, so $N \#-M \#-M$ has zero signature. Hence $\sigma(N)=2 \sigma(M)$.

REMARK. The reader will note that the only time we used Theorem 3.1 with $\chi \neq 0$ was in a case where everything could be taken smooth.

4. Examples. In the following examples we omit all calculational details. Nullity may be rather easily calculated from a projection of the link via an algorithm due to H. Seifert [17]. This is also discussed by Fox [3]. Signatures may be calculated by using Murasugi's matrix [15] or by directly finding the Seifert pairing (see [7]).

(1) Let $L$ be the link illustrated in Figure 3 (the Whitehead link [23]). One finds that $\eta(L)=1$. On the other hand, $L$ is link homotopic to a trivial link of two circles. Since the latter has nullity 2 , we see that nullity is not an invariant of link homotopy, and the inequality of Theorem 2.3 may be strict.

(2) Let $L$ and $L^{\prime}$ be the links illustrated in Figure 4. They differ only in orientation, and yet $\sigma(L)=-3$ while $\sigma\left(L^{\prime}\right)=1$. Thus link signature depends strongly upon the orientation choice. The difference is seen clearly by comparison of spanning surfaces for $L$ and $L^{\prime}$. 


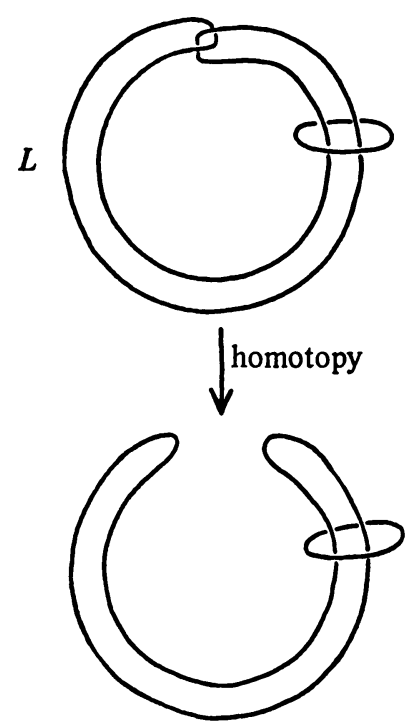

FIGURE 3
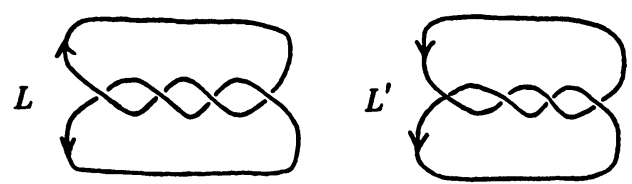

FIGURE 4

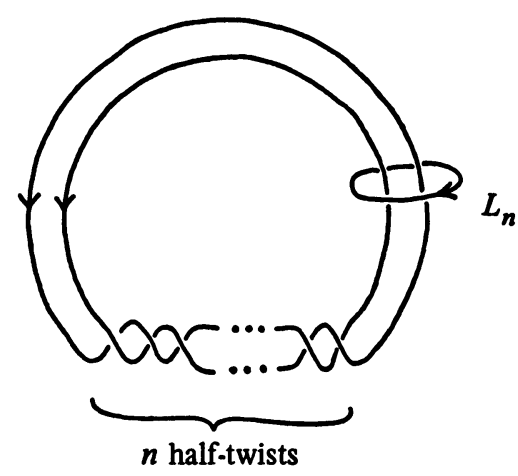

FIGURE 5

(3) For $n$ an odd, nonnegative integer, let $L_{n}$ be the link in Figure 5. We find that $\sigma\left(L_{n}\right)=2-n$. Since $S^{3}-L_{n}$ is homeomorphic to $S^{3}-L_{m}$ for any odd integers $n, m$, this gives an infinite family of links all sharing the same complement. Since they all have different signatures, no two are concordant.

This underscores the necessity of knowing the link, not merely the complement.

As a final remark, we wish to note that the approach to signature used here 
can be extended to higher order cyclic branched covers. We hope to make these higher signatures the subject of another paper.

\section{REFERENCES}

1. M. F. Atiyah and I. M. Singer, The index of elliptic operators. III, Ann. of Math. (2) 87 (1968), 546-604. MR 38 \#5245.

2. D. Erle, Die quadratische Form eines Knotens und ein Satz über Knotenmannig. faltigkeiten, J. Reine Angew. Math. 236 (1969), 174-218. MR 40 \#2106.

3. R. H. Fox, A quick trip through knot theory, Topology of 3-Manifolds and Related Topics (Proc. Univ. of Georgia Inst., 1961), Prentice-Hall, Englewood Cliffs, N. J., 1962, pp. 120-167. MR 25 \#3522.

4. F. Hosekawa, On V-polynomials of links, Osaka Math. J. 10 (1958), 273-282. MR 21 \#1606.

5. F. Hosekawa and S. Kinoshita, On the homology group of branched cyclic covering spaces of links, Osaka Math. J. 12 (1960), 331-355. MR 23 \#A2878.

6. K. Jänich and E. Ossa, On the signature of an involution, Topology 8 (1969), 27-30. MR 38 \#6613.

7. L. Kauffman, Cyclic branched covers, $O(n)$-actions, and hypersurface singularities, Thesis, Princeton Univ., Princeton, N. J., 1972. 143-160.

8. - Open books, branched covers and knot periodicity, Topology 13 (1974),

9. R. Kirby and L. Siebenmann, Codimension two locally flat imbeddings, Notices Amer. Math. Soc. 18 (1971), 983. Abstract \#71T-G196.

10. R. Kirby and L. Siebenmann (to appear).

11. H. Kneser, Die Deformationsatz der einfach zusammenhangenden Flächen, Math. Z. 25 (1926), 362-372.

12. J. W. Milnor, Infinite cyclic coverings, Conference on the Topology of Manifolds (Michigan State Univ., E. Lansing, Mich., 1967), Prindle, Weber \& Schmidt, Boston, Mass., 1968, pp. 115-133. MR 39 \#3497.

13. - Link groups, Ann. of Math. (2) 59 (1954), 177-195. MR 17, 70.

14. - Isotopy of links, Algebraic Geometry and Topology (Sympos. in Honor of S. Lefschetz), Princeton Univ. Press, Princeton, N. J., 1957, pp. 280-306. MR 19, $10 \%$.

15. K. Murasugi, On a certain numerical invariant of link types, Trans. Amer. Math. Soc. 117 (1965), 387-422. MR 30 \#1506.

16. - On the signature of links, Topology 9 (1970), 283-298. MR 41 \#6198.

17. H. Seifert, Über das Geschlecht von Knoten, Math. Ann. 110 (1934), 571-592.

18. E. H. Spanier, Algebraic topology, McGraw-Hill, New York, 1966. MR $35 \# 1007$.

19. L. Taylor, On the homology class of a topological hypersurface (to appear).

20. R. Thom, Quelques propriétés globales des variétés différentiables, Comment. Math. Helv. 28 (1954), 17-86. MR 15, 890.

21. H. F. Trotter, Homology of group systems with applications to knot theory, Ann. of Math. (2) 76 (1962), 464-498. MR 26 \#61.

22. C. T. C. Wall, Diffeomorphisms of 4-manifolds, J. London Math. Soc. 39 (1964), 131-140. MR 29 \#626.

23. J. H. C. Whitehead, $A$ certain region in Euclidean 3-space, Proc. Nat. Acad. Sci. U.S.A. 21 (1935), 364-366.

DEPARTMENT OF MATHEMATICS, UNIVERSITY OF ILLINOIS AT CHICAGO CIRCLE, CHICAGO, ILLINOIS 60680

DEPARTMENT OF MATHEMATICS, UNIVERSITY OF NOTRE DAME, NOTRE DAME, INDIANA 46556 